\title{
Effects of acrylamide in the presence of vitamin E on sperm parameters, chromatin quality, and testosterone levels in mice
}

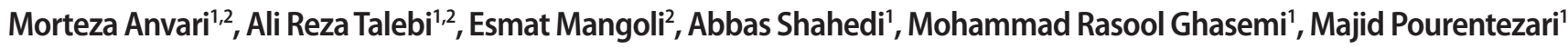 \\ ${ }^{1}$ Department of Biology and Anatomical Sciences, Shahid Sadoughi University of Medical Sciences, Yazd; ${ }^{2}$ Research and Clinical Center for Infertility, \\ Shahid Sadoughi University of Medical Sciences, Yazd, Iran
}

Objective: The present study investigated sperm chromatin quality and testosterone levels in acrylamide-treated mice and the possible protective effects of vitamin $\mathrm{E}$ on the fertility potential of spermatozoa.

Methods: Thirty-two adult male mice were divided equally into four groups. Group 1 was the control, group 2 received acrylamide (10 mg/kg, water solution), group 3 received vitamin $\mathrm{E}(100 \mathrm{mg} / \mathrm{kg}$, intraperitoneal), and group 4 received both acrylamide and vitamin E. After 35 days, spermatozoa from the right cauda epididymis were analyzed in terms of count, motility, morphology, and viability. Sperm DNA integrity and chromatin condensation were assessed by acridine orange (AO), aniline blue (AB), toluidine blue (TB), and chromomycin A3 (CMA3) staining. Results: In acrylamide-treated mice, significantly lower sperm concentration, viability, motility, and testosterone levels were found in comparison with the control and acrylamide+vitamin $\mathrm{E}$ groups $(p<0.05)$. In the vitamin $\mathrm{E}$ group, significantly more favorable sperm parameters and testosterone levels were found than in the other groups $(p<0.05)$. There were also significantly more spermatozoa with less condensed chromatin in the acrylamide-treated mice than in the other groups. Moreover, significantly more spermatozoa with mature nuclei (assessed by $A B$, $\mathrm{CMA} 3, \mathrm{AO}$, and TB staining) were present in the vitamin E group than in the control and acrylamide+vitamin E groups.

Conclusion: This study revealed the deleterious effects of acrylamide on sperm parameters and sperm chromatin quality. Vitamin $\mathrm{E}$ can not only compensate for the toxic effects of acrylamide, but also improve sperm chromatin quality in mice.

Keywords: Acrylamide; Chromatin; Mice; Spermatozoa; Testosterone; Vitamin E

\section{Introduction}

Acrylamide, which has a molecular formula of $\mathrm{CH}_{2}=\mathrm{CHCONH}_{2}$, is a white, odorless, crystalline solid that exists in naturally occurring products such as potatoes, radishes, carrots, lettuce, Chinese cabbage, onions, spinach, parsley, rice, sugar, and olives [1,2]. People are exposed to acrylamide in laboratory and factory settings, as well as in daily life via dietary intake and drinking water. In recent years, the

Received: August 11, 2019· Revised: October 11, 2019. Accepted: November 3, 2019 Corresponding author: Majid Pourentezari

Department of Biology and Anatomical Sciences, Shahid Sadoughi University of Medical Sciences, Alem Sq, Yazd 8915173143, Iran

Tel:+98-3538203410 Fax:+98-3538203414 E-mail:m.pourentezari@gmail.com

This is an Open Access article distributed under the terms of the Creative Commons Attribution Non-Commercial License (http://creativecommons.org/licenses/by-nc/4.0/) which permits unrestricted non-commercial use, distribution, and reproduction in any medium, provided the original work is properly cited. toxicity of acrylamide has been the subject of many studies [3,4]. It has been reported that the acrylamide exerts mutagenic and carcinogenic actions through oxidation to glycidamide by the cytochrome P450 2E1 protein [5]. Acrylamide is, furthermore, considered to be a toxicant of the male reproductive system in animals, both morphologically and genetically [6]. Our previous study found that acrylamide had detrimental effects on sperm motility, membrane integrity, and sperm vitality [7]. It should also be noted that acrylamide induces histopathological lesions such as the formation of multinucleated giant cells and vacuolation, and increases apoptosis in seminiferous tubules [8]. Oxidative stress may be an important harmful effect of acrylamide on epididymal spermatozoa. Elevated reactive oxygen species (ROS) levels may play an integral role in the pathogenesis of sperm chromatin/DNA damage [9]. High levels of ROS in the semen can trigger sperm dysfunction, sperm DNA damage, and 
reduced male reproductive potential. Therefore, it has been suggested that dietary antioxidant supplements can have beneficial effects on sperm function and chromatin integrity [10].

Vitamin $\mathrm{E}$ is a major antioxidant with the capacity to interrupt lipid peroxidation reactions [11]. Moreover, this supplement is considered to be a free radical scavenger that also enhances the production of other free radical scavengers [12]. The use of antioxidants increases sperm quality and DNA integrity [13]. Since compensating for the harmful effects of acrylamide on sperm nuclear structure is of paramount importance, the aim of the present study was to evaluate sperm chromatin condensation and testosterone levels after administration of vitamin $\mathrm{E}$ to acrylamide-treated mice.

\section{Methods}

\section{Animals and treatments}

Thirty-two adult male Syrian mice ( $35 \mathrm{~g}, 10$ weeks old) were assigned to four groups, each comprising eight mice. The mice in group 1 were fed a basal diet and served as the control group, group 2 received the basal diet and acrylamide (Merck, Darmstadt, Germany; $10 \mathrm{mg} / \mathrm{kg}$, water solution) [7]; group 3 received the basal diet and vitamin E (Osvah Pharmaceutical, Tehran, Iran; 100 mg/kg, intraperitoneal) [14]; and group 4 received the basal diet, acrylamide, and vita$\min E$ for 35 days (almost equal to the duration of a cycle of spermatogenesis). They were kept in cages in a controlled environment with a temperature range of $25^{\circ} \mathrm{C} \pm 3^{\circ} \mathrm{C}$ and mean relative humidity of $50 \% \pm 5 \%$. A chemical analysis indicated that acrylamide was stable in water for at least 1 week, so the solutions were prepared and changed weekly. The body weight of the mice and their water consumption were monitored throughout the course of the experiment. We followed the recommendations set forth by Institutional Animal Care and Use Committee of Shahid Sadoughi University of Medical Sciences for the maintenance, handling, treatment, and killing of the laboratory animals.

\section{Sampling}

On the last day, blood samples were taken from the animals for testosterone analysis. The mice were then killed by cervical dislocation and the dissected epididymis of each animal was transferred into 1 $\mathrm{mL}$ of pre-warmed Ham's F10 medium and cut into small slices for the sperm cells to swim out into the medium. The samples were incubated at $37^{\circ} \mathrm{C}$ for 30 minutes [15].

\section{Sperm analysis}

The sperm samples were evaluated according to World Health Organization (WHO) guidelines [16]. Sperm parameters including count $\left(\times 10^{6}\right)$, motility, morphology, and viability (\%) were analyzed for each animal. For the evaluation of sperm count and motility, we used a Makler chamber. The percentage of sperm motility was analyzed for the following motion patterns: progressive (grades A and B), non-progressive (grade C) and immotile (grade D) spermatozoa [17]. Eosin-nigrosin staining was used to assess sperm viability according to the WHO protocol $[16,17]$. To evaluate sperm with normal morphology, the slides were stained with the Papanicolaou stain. A total of 200 spermatozoa per sample were counted and the percentages of normal morphology were recorded [18].

\section{Sperm chromatin/DNA evaluation}

Chromatin condensation and DNA integrity assessments were carried out using cytochemical techniques including acridine orange (AO), toluidine blue (TB), aniline blue (AB), and chromomycin $A 3$ (CMA3). All dyes and chemicals were purchased from Sigma Aldrich (St. Louis, MO, USA).

\section{1) $\mathrm{AO}$ test}

$\mathrm{AO}$ is a metachromatic fluorescent probe used to demonstrate the degree of sperm nuclear DNA susceptibility to in situ acid-induced denaturation by distinguishing between native double-stranded DNA (green fluorescence) and denatured single-stranded DNA (red or yellow fluorescence). Air-dried smears were fixed in Carnoy's solution (methanol/glacial acetic acid, $3: 1$ ) at $4^{\circ} \mathrm{C}$ for at least 2 hours. Each sample was stained with freshly prepared AO $(0.19 \mathrm{mg} / \mathrm{mL}$ in Mcllvaine phosphate-citrate buffer; $\mathrm{pH}$ 4) for 10 minutes. Ultimately, the smears were assessed on the same day using fluorescence microscopy (Zeiss, Jena, Germany) with a 460-nm filter [19].

\section{2) TB staining}

$T B$ is a metachromatic dye used to determine both the quality and the quantity of sperm nuclear chromatin condensation and DNA fragmentation via binding to phosphate groups of DNA strands. Briefly, air-dried sperm smears were fixed with fresh $96 \%$ ethanol-acetone $(1: 1)$ at $4^{\circ} \mathrm{C}$ for 30 minutes and then hydrolyzed in $0.1 \mathrm{~N} \mathrm{HCl}$ at $4^{\circ} \mathrm{C}$ for 5 minutes. After washing, the slides were stained with $0.05 \%$ TB in 50\% citrate phosphate for 10 minutes at room temperature. In each sample, at least 200 spermatozoa were counted under light microscopy using $\times 100$ eyepiece magnification [7].

\section{3) $A B$ staining}

$A B$ selectively stains lysine-rich histones and is used to detect spermatozoa with excessive histones in the chromatin structure. To perform $A B$ staining, air-dried smears were fixed in $3 \%$ buffered glutaraldehyde in $0.2 \mathrm{M}$ phosphate buffer ( $\mathrm{pH} 7.2$ ) for 30 minutes at room temperature. Each smear was stained with $5 \%$ aqueous $A B$ stain in $4 \%$ acetic acid (pH 3.5) for 7 minutes. Under light microscopy, 200 
spermatozoa were counted in different areas of each slide using $\times 100$ eyepiece magnification [20].

\section{4) $C M A 3$ staining}

This fluorescent probe is used to detect sperm cells with protamine deficiency. To carry out the staining, sperm smears were fixed in methanol/glacial acetic acid (3:1) at $4^{\circ} \mathrm{C}$ for 10 minutes, and then were treated with $100 \mu \mathrm{L}$ of CMA3 solution $(0.25 \mathrm{mg} / \mathrm{mL}$ in Mcllvaine buffer at $\mathrm{pH} 7.0$, containing $10 \mathrm{mM} \mathrm{MgCl}$ ) for 20 minutes in a darkroom. The slides were rinsed in buffer and mounted with buffered glycerol. The spermatozoa were evaluated by fluorescence microscopy (Zeiss, Oberkochen, Germany). Two sperm populations were identified: sperm cells with bright green fluorescent heads (abnormal chromatin packaging) and those with non-fluorescent heads (normal chromatin packaging) [19]. This study was approved by the Ethics Committee of Shahid Sadoughi University of Medical Sciences in Yazd, Iran. All the procedures were conducted carefully to meet national laboratory standards.

\section{Statistical analysis}

Data normalization was performed using the Kolmogorov-Smirnov test. All data are presented as mean \pm standard deviation. One-way analysis of variance was applied to evaluate the significance of differences among the groups, and the Tukey post-hoc test was used to evaluate the differences between pairs of groups. A $p$-value $\leq 0.05$ was considered to indicate statistical significance. Statistical analysis was conducted using IBM SPSS ver. 20.0 (IBM Corp., Armonk, NY, USA).

\section{Results}

Table 1 presents the mean values and results of the statistical analysis of various sperm parameters in the four groups. The sperm count, sperm motility grades ( $A, B, C$, and $D)$, sperm morphology, and viability were significantly different among the groups $(p<0.05)$. Table 1 also shows the results of the analysis of serum testosterone levels in the four groups. The acrylamide-treated mice showed significantly lower sperm concentration, viability, motility, and testosterone hormone levels than the control and acrylamide+vitamin E groups $(p<0.05)$. In the vitamin E group, significantly more favorable sperm parameters and testosterone levels were found than in the other groups $(p<0.05)$.

Table 2 presents the results of the sperm chromatin and DNA integrity evaluations. For $A B$ staining, the percentages of unstained or pale blue-stained (normal spermatozoa) and dark blue-stained (abnormal spermatozoa) were reported. In TB staining, the chromatin quality of sperm was assessed according to metachromatic staining of sperm heads with the following scores: 0 , light blue (good chromatin); 1 , dark blue (mildly abnormal chromatin); 2 , violet; and 3, purple (severely abnormal chromatin). Therefore, all spermatozoa with scores of 1,2 , and 3 were considered to be $\mathrm{TB}^{+}$or sperm cells with abnormal chromatin, whereas score 0 spermatozoa were considered as $\mathrm{TB}^{-}$or

Table 1. Results of sperm analysis and serum testosterone levels in mice in different groups

\begin{tabular}{|c|c|c|c|c|c|}
\hline Variable & Control group & Acrylamide group & Vitamin E group & $\begin{array}{c}\text { Acrylamide+vitamin } \\
\text { E group }\end{array}$ & $p$-value \\
\hline Count $\left(\times 10^{6}\right)$ & $110 \pm 17.49$ & $90.12 \pm 10$ & $132.5 \pm 19.11$ & $98 \pm 11.07$ & $\begin{array}{l}0.000^{\mathrm{a}, \mathrm{b})} \\
0.033^{\mathrm{c})}\end{array}$ \\
\hline Rapid motility (\%, grade A) & $20.75 \pm 3.01$ & $16.25 \pm 3.49$ & $28.75 \pm 3.32$ & $18.75 \pm 1.31$ & $0.01^{\text {a) }}$ \\
\hline Slow motility (\%, grade B) & $23.25 \pm 4.86$ & $14.37 \pm 3.66$ & $27.62 \pm 4.62$ & $23.25 \pm 5.77$ & $\begin{array}{l}0.000^{\mathrm{a})} \\
0.006^{\mathrm{d})}\end{array}$ \\
\hline Non-progressive motility (\%, grade C) & $32 \pm 4.37$ & $18.12 \pm 5.54$ & $26 \pm 7.94$ & $24.5 \pm 7.23$ & $0.001^{\mathrm{d})}$ \\
\hline Immotile sperm (\%, grade D) & $24 \pm 4.276$ & $50 \pm 7.69$ & $17.62 \pm 5.99$ & $35.5 \pm 8.45$ & $0.000^{a, b, d, e)}$ \\
\hline Total motility (\%, grades $\mathrm{A}, \mathrm{B}$, and $\mathrm{C}$ ) & $76 \pm 4.27$ & $50 \pm 7.69$ & $82.37 \pm 5.99$ & $64 \pm 1.08$ & $\begin{array}{l}0.000^{\mathrm{a}, \mathrm{b}, \mathrm{d})} \\
0.023^{\mathrm{f})} \\
0.006^{\mathrm{e})}\end{array}$ \\
\hline Normal morphology & $75.87 \pm 6.72$ & $68.5 \pm 2.97$ & $83.75 \pm 7.38$ & $74.5 \pm 5.73$ & $\begin{array}{l}0.000^{a)} \\
0.039^{b)}\end{array}$ \\
\hline Viability (\%) & $78.12 \pm 5.08$ & $66.5 \pm 5.55$ & $85.5 \pm 5.72$ & $73.75 \pm 8.11$ & $\begin{array}{l}0.000^{\mathrm{a})} \\
0.001^{\mathrm{b}, \mathrm{d})}\end{array}$ \\
\hline Testosterone level (ng/dL) & $3.25 \pm 1.58$ & $1.35 \pm 1.04$ & $6.08 \pm 2.49$ & $7.92 \pm 3$ & $\begin{array}{l}0.001^{\mathrm{a})} \\
0.000^{\mathrm{e}} \\
0.002^{\mathrm{f}}\end{array}$ \\
\hline
\end{tabular}

Values are presented as mean \pm standard deviation.

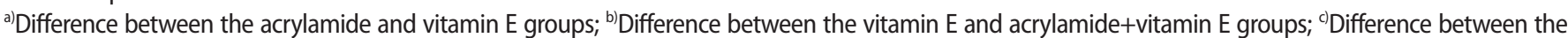
control and vitamin E groups; ${ }^{\text {d) }}$ Difference between the control and acrylamide groups; ${ }^{\mathrm{e})}$ Difference between the acrylamide and acrylamide+vitamin $\mathrm{E}$ groups; ${ }^{\text {f) }}$ Difference between the control and acrylamide+vitamin E groups. 
Table 2. Results of sperm chromatin and DNA evaluation in different groups

\begin{tabular}{|c|c|c|c|c|c|}
\hline Variable & Control group & Acrylamide group & Vitamin E group & $\begin{array}{c}\text { Acrylamide+vitamin } \mathrm{E} \\
\text { group }\end{array}$ & $p$-value \\
\hline $\mathrm{AO}$ & $8.4 \pm 3.0$ & $12.6 \pm 3.0$ & $5.0 \pm 1.6$ & $8.1 \pm 1.8$ & $\begin{array}{l}0.000^{\mathrm{a})} \\
0.006^{\mathrm{b})} \\
0.010^{\mathrm{c}}\end{array}$ \\
\hline TB & $20.5 \pm 2.4$ & $23.8 \pm 6.2$ & $17.9 \pm 3.2$ & $20.4 \pm 3.3$ & $0.041^{\mathrm{a})}$ \\
\hline$A B$ & $22.5 \pm 4.7$ & $28.8 \pm 4.3$ & $14.6 \pm 4.4$ & $21.38 \pm 4.4$ & $\begin{array}{l}0.000^{\mathrm{a})} \\
0.009^{\mathrm{d})} \\
0.016^{\mathrm{b})} \\
0.032^{\mathrm{e})}\end{array}$ \\
\hline CMA3 & $3.3 \pm 1.7$ & $22.13 \pm 4.1$ & $1.4 \pm 1.9$ & $4.8 \pm 1.4$ & $0.000^{a, b), c)}$ \\
\hline
\end{tabular}

Values are presented as mean \pm standard deviation.

$A O$, acridine orange; $T B$, toluidine blue; $A B$, aniline blue; $C M A 3$, chromomycin $A 3$.

${ }^{a}$ )Difference between the acrylamide and vitamin E groups; ${ }^{b}$ Difference between the acrylamide and acrylamide+vitamin $\mathrm{E}$ groups; ${ }^{\mathrm{c}}$ Difference between the control and acrylamide groups; ${ }^{\text {d) }}$ Difference between the control and vitamin E groups; ${ }^{\text {e) }}$ Difference between the vitamin E and acrylamide+vitamin $\mathrm{E}$ groups.

spermatozoa with normal chromatin. For AO testing, the percentages of green (normal double-stranded DNA) and orange/red (abnormally denatured DNA) fluorescent spermatozoa per sample were calculated. In CMA3 staining, bright yellow-stained chromomycin-reacted spermatozoa $\left(\mathrm{CMA3}^{+}\right)$and yellowish green-stained non-reacted spermatozoa (CMA3-) were considered as the abnormal and normal forms, respectively.

Significantly more spermatozoa with less condensed chromatin were detected in the acrylamide-treated mice than in the other groups $(p<0.05)$. Furthermore, significantly more spermatozoa with mature nuclei (assessed by $A B, C M A 3, A O$, and TB) were found in the vitamin $\mathrm{E}$ group than in the control and acrylamide+vitamin $\mathrm{E}$ groups $(p<0.05)$.

\section{Discussion}

ROS appear to play a crucial role in the generation of sperm chromatin damage [21]. In vitro and in vivo studies have demonstrated a beneficial effect of antioxidant supplements in protecting sperm chromatin from oxidative stress. This fact has led andrologists to treat infertile men with antioxidant supplements $[12,13]$. To our knowledge, the effect of antioxidants in protecting sperm from acrylamideinduced damage has not yet been established. Accordingly, the purpose of this study was to investigate the beneficial effects of vitamin E on sperm parameters, chromatin condensation, and blood testosterone levels in acrylamide-treated mice.

Previous studies have shown consistent results regarding the effects of acrylamide on sperm parameters. Yang et al. [22] found that acrylamide treatment in rats led to a significant reduction in cauda epididymal sperm concentration and an increase in the percentage of spermatozoa with abnormal morphology. Reduced serum folliclestimulating hormone, luteinizing hormone, and testosterone hor- mone concentrations were also found in the serum of acrylamidetreated rats, leading the researchers to conclude that acrylamide can impair the viability of Leydig cells, thereby diminishing the rate of spermatogenesis [22].

In a previous study, we evaluated serum parameters and serum testosterone levels in acrylamide-treated mice in comparison with controls [7]. Our findings showed poorer parameters in acrylamidetreated mice than in control animals. Furthermore, significantly lower serum testosterone concentrations were found in acrylamide-treated animals than in controls $(p<0.001)$ [7]. To explain the possible mechanism of harmful effects of acrylamide on other sperm parameters such as motility and viability, it should be noted that acrylamide can induce the production of free radicals such as ROS, which trigger the peroxidation of polyunsaturated fatty acids in the sperm membrane [23]. This may consequently lead to the destruction of sperm mitochondrial function, resulting in sperm adenosine triphosphate depletion and ultimately a reduction in sperm viability and motility [24]. We also consider that the reduced sperm motility and viability induced by acrylamide may result from the ability of this toxicant to induce oxidative stress.

An intriguing finding of our study was the improvement of sperm viability and motility in the vitamin $\mathrm{E}$ group when compared with the control animals. This effect of vitamin $E$ might be due to its antioxidant role against oxidative stress induced by acrylamide. Interestingly, we also found that in the acrylamide+vitamin E group, vitamin E significantly ameliorated the acrylamide-induced decrease in sperm viability and motility. It is generally accepted that vitamin E or alpha-tocopherol is a powerful biological antioxidant that protects cells from the harmful effects of free radicals and ROS [25]. Some studies have proposed that through its capacity to limit the production of free radicals, vitamin $\mathrm{E}$ can prevent or delay the development of chronic diseases [25]. In addition, it has been shown that vitamin $E$ 
plays a role in immune function, DNA repair, and specific metabolic processes [26]. It plays an important protective role in preventing the production of lipid peroxides by scavenging free radicals, which are toxic for biological membranes [11,12]. Therefore, it is speculated that by improving the activity of the sperm defense antioxidant system, including glutathione peroxidase, superoxide dismutase, and catalase, vitamin E improves sperm viability and motility. With regard to sperm morphology, we observed significantly higher levels of morphological anomalies in rats treated with acrylamide. A pertinent study documented the potential of ROS generation for inducing abnormal sperm morphology [27]. Nonetheless, Cao et al. [28] demonstrated that increased oxidative stress and reduced enzymatic and non-enzymatic antioxidant levels in Leydig cells played a crucial role in inducing impaired spermatogenesis and, consequently, a significant reduction in epididymal sperm count. It has also been determined that, as an effective antioxidant, vitamin $\mathrm{E}$ can protect rabbit testes against lipid peroxidation [29]. According to our findings, the improvements in sperm count and normal sperm morphology in the vitamin $\mathrm{E}$ group, compared with the control group, may have been due to the antioxidant effects of vitamin E. In line with our study, Sonmez et al. [30] reported evidence that chronic administration of homocysteine as an oxidant similar to acrylamide had a harmful effect on the epididymal sperm characteristics of male rats and that administration of vitamin E could prevent those complications. Moreover, vitamin E was found to trigger elevated plasma antioxidant enzyme activity and to improve testosterone levels, sperm motility, and sperm count in male rats.

Routine findings from semen evaluations, such as sperm concentration, morphology, and motility, may fail to identify defects in sperm chromatin structure. The evaluation of sperm chromatin damage appears to be a useful way to assess male fertility potential, as sperm chromatin abnormalities or DNA damage may result in male infertility. Among the various sperm chromatin anomalies that can be present in the male gamete, DNA fragmentation is the most frequent, particularly in infertile men $[19,31]$. It is generally accepted that a clear relationship exists between sperm chromatin/DNA damage and reproductive outcomes. Furthermore, sperm quality plays a vital role in early embryonic growth and pregnancy outcomes [32].

TB staining enables the visualization of spermatozoa with fragmented DNA and/or abnormal chromatin structure. It is used as a routine test for sperm DNA integrity because it is an easy-to-run and inexpensive method that is complementary to standard semen parameters [33]. According to our data from $\mathrm{TB}^{-}$staining, although a difference was observed in the percentage of TB-reacted spermatozoa among the groups, it was not statistically significant. Moreover, the $\mathrm{AO}$ test showed a notably lower percentage of normal sperm in the acrylamide group than in the control, vitamin E, and vitamin
E+acrylamide groups. It can thus be concluded that acrylamide causes the denaturation of sperm DNA strands and vitamin E diminishes this effect. For comparative purposes, however, we failed to find any similar research investigating the effects of acrylamide on sperm DNA denaturation. AB staining detects the presence of histones, and indirectly shows the presence of lower amounts of protamine in the sperm nucleus. In this study, we observed a higher proportion of spermatozoa with residual histones in the acrylamide group than in the control group, but no significant difference was found between the vitamin $\mathrm{E}$ and vitamin $\mathrm{E}+$ acrylamide groups and the control mice. Moreover, we identified a higher proportion of $\mathrm{CMA}^{+}$(abnormal chromatin packaging) spermatozoa in acrylamidetreated mice than in the other three groups. As noted above, the CMA3 test reveals protamine deficiency in the process of sperm chromatin condensation [34]; therefore, it can be inferred that acrylamide contributes to sperm protamine deficiency in mice. It should be noted that our findings are novel with regard to the use of cytochemical staining to determine residual histone and protamine deficiency in the spermatozoa of acrylamide-treated laboratory animals. Acrylamide was found to exert negative effects on sperm parameters and sperm chromatin quality in mice. Vitamin E not only compensated for the toxic effects of acrylamide on sperm parameters, but also improved sperm chromatin quality in mice.

\section{Conflict of interest}

No potential conflict of interest relevant to this article was reported.

\section{Acknowledgments}

The authors thank all their colleagues at Shahid Sadoughi University of Medical Sciences (Yazd, Iran) for their cooperation.

\section{ORCID}

$\begin{array}{ll}\text { Morteza Anvari } & \text { https://orcid.org/0000-0003-3114-9513 } \\ \text { Ali Reza Talebi } & \text { https://orcid.org/0000-0003-4883-3020 } \\ \text { Esmat Mangoli } & \text { https://orcid.org/0000-0001-6206-7831 } \\ \begin{array}{ll}\text { Abbas Shahedi } & \text { https://orcid.org/0000-0002-9445-3806 } \\ \text { Mohammad Rasool Ghasemi } \\ \text { https://orcid.org/0000-0002-5106-2768 }\end{array} \\ \begin{array}{c}\text { Majid Pourentezari } \\ \text { https://orcid.org/0000-0001-7549-4672 }\end{array}\end{array}$

\section{Author contributions}

Conceptualization: MA, ART. Data curation: MP. Formal analysis: EM. 
Methodology: MA, ART. Writing-original draft: MP. Writing-review \& editing: $A S, M R G$.

\section{References}

1. Kafouris D, Stavroulakis G, Christofidou M, lakovou X, Christou E, Paikousis $L$, et al. Determination of acrylamide in food using a UPLC-MS/MS method: results of the official control and dietary exposure assessment in Cyprus. Food Addit Contam Part A Chem Anal Control Expo Risk Assess 2018;35:1928-39.

2. Duke TJ, Ruestow PS, Marsh GM. The influence of demographic, physical, behavioral, and dietary factors on hemoglobin adduct levels of acrylamide and glycidamide in the general U.S. population. Crit Rev Food Sci Nutr 2018;58:700-10.

3. Tabeshpour J, Mehri S, Abnous K, Hosseinzadeh H. Neuroprotective effects of thymoquinone in acrylamide-induced peripheral nervous system toxicity through MAPKinase and apoptosis pathways in rat. Neurochem Res 2019;44:1101-12.

4. Bao W, Cao C, Li S, Bo L, Zhang M, Zhao X, et al. Metabonomic analysis of quercetin against the toxicity of acrylamide in rat urine. Food Funct 2017;8:1204-14.

5. Markovic J, Stosic M, Kojic D, Matavulj M. Effects of acrylamide on oxidant/antioxidant parameters and CYP2E1 expression in rat pancreatic endocrine cells. Acta Histochem 2018;120:73-83.

6. Sun J, Li M, Zou F, Bai S, Jiang X, Tian L, et al. Protection of cyanidin-3-O-glucoside against acrylamide- and glycidamide-induced reproductive toxicity in leydig cells. Food Chem Toxicol 2018;119:268-74.

7. Pourentezari M, Talebi A, Abbasi A, Khalili MA, Mangoli E, Anvari $M$. Effects of acrylamide on sperm parameters, chromatin quality, and the level of blood testosterone in mice. Iran J Reprod Med 2014;12:335-42.

8. Hasanin NA, Sayed NM, Ghoneim FM, Al-Sherief SA. Histological and ultrastructure study of the testes of acrylamide exposed adult male albino rat and evaluation of the possible protective effect of vitamin E intake. J Microsc Ultrastruct 2018;6:23-34.

9. Bui AD, Sharma R, Henkel R, Agarwal A. Reactive oxygen species impact on sperm DNA and its role in male infertility. Andrologia 2018;50:e13012.

10. Amidi F, Pazhohan A, Shabani Nashtaei M, Khodarahmian M, Nekoonam S. The role of antioxidants in sperm freezing: a review. Cell Tissue Bank 2016;17:745-56.

11. Zare S, Hossein Dabbaghmanesh M, Noorafshan A, Koohpeyma F, Bakhshayeshkaram M, Montazeri-Najafabady N. Protective effect of vitamin $\mathrm{E}$ and vitamin C alone and in combination on testicular damage induced by sodium metabisulphite in rats: a stereological study. Andrologia 2019;51:e13193.
12. Wang CY, Zhang JJ, Duan P. Antagonistic effect of vitamin E on di-2-ethylhexyl phthalate-induced reproductive toxicity in male rats. Zhonghua Nan Ke Xue 2018;24:589-95.

13. Gual-Frau J, Abad C, Amengual MJ, Hannaoui N, Checa MA, Ribas-Maynou J, et al. Oral antioxidant treatment partly improves integrity of human sperm DNA in infertile grade I varicocele patients. Hum Fertil (Camb) 2015;18:225-9.

14. Gavazza M, Catala A. The effect of alpha-tocopherol on the lipid peroxidation of mitochondria and microsomes obtained from rat liver and testis. Mol Cell Biochem 2001;225:121-8.

15. Bandegi L, Anvari M, Vakili M, Khoradmehr A, Mirjalili A, Talebi AR. Effects of antidepressants on parameters, melondiadehyde, and diphenyl-2-picryl-hydrazyl levels in mice spermatozoa. Int J Reprod Biomed (Yazd) 2018;16:365-72.

16. World Health Organization. WHO laboratory manual for the examination of human semen and sperm-cervical mucus interaction. Cambridge: Cambridge University Press; 1999.

17. Momeni HR, Eskandari N. Effect of vitamin E on sperm parameters and DNA integrity in sodium arsenite-treated rats. Iran J Reprod Med 2012;10:249-56.

18. Sabour M, Khoradmehr A, Kalantar SM, Danafar AH, Omidi M, Halvaei I, et al. Administration of high dose of methamphetamine has detrimental effects on sperm parameters and DNA integrity in mice. Int J Reprod Biomed (Yazd) 2017;15:161-8.

19. Pourentezari M, Talebi AR, Mangoli E, Anvari M, Rahimipour M. Additional deleterious effects of alcohol consumption on sperm parameters and DNA integrity in diabetic mice. Andrologia 2016;48:564-9.

20. Mangoli E, Talebi AR, Anvari M, Pourentezari M. Effects of experimentally-induced diabetes on sperm parameters and chromatin quality in mice. Iran J Reprod Med 2013;11:53-60.

21. Sharma RK, Agarwal A. Role of reactive oxygen species in male infertility. Urology 1996;48:835-50.

22. Yang HJ, Lee SH, Jin Y, Choi JH, Han DU, Chae C, et al. Toxicological effects of acrylamide on rat testicular gene expression profile. Reprod Toxicol 2005;19:527-34.

23. Zhang JX, Yue WB, Ren YS, Zhang CX. Enhanced fat consumption potentiates acrylamide-induced oxidative stress in epididymis and epididymal sperm and effect spermatogenesis in mice. Toxicol Mech Methods 2010;20:75-81.

24. Das J, Ghosh J, Manna P, Sinha M, Sil PC. Taurine protects rat testes against $\mathrm{NaAsO}(2)$-induced oxidative stress and apoptosis via mitochondrial dependent and independent pathways. Toxicol Lett 2009;187:201-10.

25. Fiedor J, Burda K. Potential role of carotenoids as antioxidants in human health and disease. Nutrients 2014;6:466-88.

26. Baran OP, Tunik S, Akkoc H, Devieci E, Ayaz E, Soker S, et al. The 
prophylactic effects of folic acid and vitamin E against valproic acid during fetal thymus development: an ultrastructural study. Int J Morphol 2011;29:1093-8.

27. Venkatesh A, Singh G, Gupta NP, Kumar R, Deecaraman M, Dada R. Correlation of sperm morphology and oxidative stress in infertile men. Iran J Reprod Med 2009;7:29-34.

28. Cao L, Leers-Sucheta S, Azhar S. Aging alters the functional expression of enzymatic and non-enzymatic anti-oxidant defense systems in testicular rat Leydig cells. J Steroid Biochem Mol Biol 2004;88:61-7.

29. Kalender S, Uzun FG, Demir F, Uzunhisarcıkli M, Aslanturk A. Mercuric chloride-induced testicular toxicity in rats and the protective role of sodium selenite and vitamin E. Food Chem Toxicol 2013;55:456-62.

30. Sonmez M, Yuce A, Turk G. The protective effects of melatonin and vitamin $\mathrm{E}$ on antioxidant enzyme activities and epididymal sperm characteristics of homocysteine treated male rats. Reprod Toxicol 2007;23:226-31.
31. Tamburrino L, Marchiani S, Montoya M, Elia Marino F, Natali I, Cambi $M$, et al. Mechanisms and clinical correlates of sperm DNA damage. Asian J Androl 2012;14:24-31.

32. Rahimipour M, Talebi AR, Anvari M, Abbasi Sarcheshmeh A, Omidi M. Saccharin consumption increases sperm DNA fragmentation and apoptosis in mice. Iran J Reprod Med 2014;12:307-12.

33. Talebi AR, Mangoli E, Nahangi $H$, Anvari M, Pourentezari $M$, Halvaei I. Vitamin $C$ attenuates detrimental effects of diabetes mellitus on sperm parameters, chromatin quality and rate of apoptosis in mice. Eur J Obstet Gynecol Reprod Biol 2014;181: 32-6.

34. Khalili MA, Nazari S, Dehghani-Firouzabadi R, Talebi A, Baghazadeh-Naeini S, Sadeghian-Nodoshan F, et al. Comparing the roles of sperm chromatin integrity and apoptosis in intrauterine insemination outcomes of couples with mild male and female factor infertility. J Reprod Infertil 2014;15:35-40. 